\title{
New Targets and New Treatments: Recent Advances in the Treatment of Musculoskeletal Disease
}

\author{
Stuart H. Ralston ${ }^{1} \cdot$ Rene Rizzoli $^{2}$
}

Received: 2 March 2016/Accepted: 3 March 2016/Published online: 17 March 2016

(C) Springer Science+Business Media New York 2016

Over the past two decades, huge strides have been made in understanding the genes and molecular pathways that contribute to the pathogenesis of bone and muscle diseases. As a result of these advances, several new molecular targets have been identified for drug design and this knowledge is now being harnessed by the pharmaceutical industry to develop new drugs for the treatment of sarcopenia and metabolic bone diseases like osteoporosis, hypophosphataemic rickets, and hypophosphatasia. The current issue of Calcified Tissue International and Musculoskeletal Research highlights some of the most notable advances that have occurred in this fast developing area over recent years.

The special issue begins with the review article by Morley which provides a comprehensive update on the pathophysiology of sarcopenia documenting the key cytokines and other regulatory molecules that regulate muscle function. Subsequently, Morley goes on to review the pharmacological agents that have already been investigated in clinical trials while also giving an insight into the future targets for therapeutic intervention. The review of the FGF-23 and Klotho pathway by Fukomoto similarly provides an excellent overview of the role that this network of hormones and receptors plays in the pathophysiology of hypophosphataemic rickets, tumoral calcinosis, and metabolic bone disease-associated chronic kidney disease

Stuart H. Ralston

stuart.ralston@ed.ac.uk

1 Centre for Genomic and Experimental Medicine, Institute of Genetics and Molecular Medicine, University of Edinburgh, Edinburgh, UK

2 Geneva University Hospitals and Faculty of Medicine, Geneva, Switzerland
(CKD-MBD). The results of clinical trials with anti-FGF23 antibodies are reviewed and finally future prospects for modulating the FGF-23 pathway in hypophosphataemic rickets, tumoral calcinosis, and CKD-MDB are discussed.

Next, Nemeth and Goodman provide a fascinating insight into the progress (and setbacks) that have been encountered in targeting the calcium sensing receptor (CaSR) for the treatment of bone diseases. The article begins with an account of the physiology of the CaSR and its downstream signalling pathways followed by a brief account of the genetic diseases associated with CaSR mutations. Subsequently, the clinical studies with calcimimetics (drugs which mimic the effects of calcium, thereby reducing PTH secretion) and calcilytics (drugs which act as antagonists at the CaSR and increase PTH secretion) are reviewed. As the authors acknowledge, the initial hope that calcilytics might prove to be useful as anabolic agents were not fulfilled, although the calcimimetics have now an established role in the treatment of primary hyperparathyroidism and tertiary hyperparathyroidism associated with renal bone disease.

The next two review articles focus on the development of new anabolic drugs for the treatment of osteoporosis. The first of these, authored by Esbrit and colleagues, provides an account of the physiology of parathyroid hormone-related protein (PTHrP) and the drugs that have been developed to target this pathway. Of special interest is a particular focus on abaloparatide which has recently shown promising results in phase III clinical trials. The second article by Appelman-Dijkstra and Papapoulos documents the progress that has been made in targeting the sclerostin pathway in the treatment of osteoporosis in a review which encompasses the underlying physiology and preclinical studies as well as the results of the phase I and phase II clinical studies. 
The article by Duong and colleagues provides a comprehensive account of the genetics, biology, pharmacology, and clinical trial data underlying the development of the Cathepsin $\mathrm{K}$ inhibitor odanacatib as a new treatment for osteoporosis. Although the results of the pivotal LOFT trial remain to be fully published, preliminary results presented in abstract form and summarised in the review demonstrate that odanacatib has robust anti-fracture efficacy and may hopefully be available for clinical use in the non-too-distant future.

The final paper by Millan and Whyte reviews the remarkable developments that have been made in the treatment of hypophosphatasia $-\mathrm{a}$ rare genetic condition caused by loss of function mutations in the tissue nonspecific alkaline phosphatase gene (TNSALP). The review provides a detailed and authoritative bench-to-bedside account of the clinical features of the disease, biology of the alkaline phosphatase enzyme, and the results that have emerged from the preclinical and clinical development programmes of alkaline phosphatase replacement therapy.

It has been a great pleasure to bring this unique set of articles together into this special "Targets and Treatments" issue of the journal. We very much hope that the readers of Calcified Tissue International and Musculoskeletal Research will find the reviews as enjoyable and informative to read as we have. 